\title{
Short cuts
}

\author{
What's new in the other general journals
}

\section{Stopping violations of human rights in Haiti needs more action}

Since the democratically elected Haitian president Jean Bertrand Aristide was overthrown in February 2004, reports of human rights violations have ranged from several hundred to more than 100000 affected people. Nobody, including the interim government and the United Nations, has provided believable estimates of the number of assaults or the identity of perpetrators.

A recent survey of 1260 randomly selected households in the wider area of Port-au-Prince included more than 5700 individuals and looked at human rights violations between February 2004 and December 2005. The researchers estimated that in the studied area over that period about 8000 murders took place, along with about 35000 sexual assaults of women, 21000 physical assaults, 11000 kidnappings or extrajudicial detentions, and 136000 threats of death, physical injury, or sexual violence.

Almost half of the murders seemed to have been committed for political purposes. More than half of sexual assaults were on girls younger than 18 years, and one in ten "restaveks" (girls who work as unpaid domestic servants) had been sexually assaulted. Anecdotal evidence suggests that many people whose human rights were violated in the spring of 2004 fled the area. If true, this would mean that the study underestimates the extent of violence.

An editorial (p 816) points out that 14\% of participants in the survey claimed to have been threatened by foreign soldiers, including those in the UN peacekeeping forces that have been intermittently present in the area for 15 years. Kofi Annan's stand against exploitative behaviour by UN peacekeepers needs to be followed by stronger action to avoid adding to people's suffering.

Lancet DOI:10.1016/S0140-6736(06)69211-8

\section{Contact lens solution withdrawn from market after epidemiological investigation}

Although the risk of microbial keratitis is 80 times higher in people who wear contact lenses than in people who don't, fungal keratitis is relatively rare, accounting for about $5 \%$ of keratitis cases among people who wear lenses. In February 2006 in Singapore and Hong Kong, clusters of

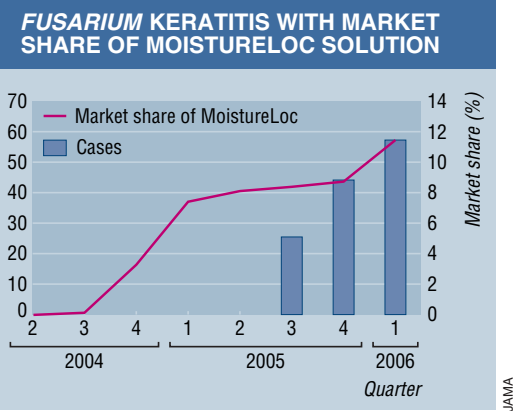

people who wore lenses were reported to have Fusarium keratitis. Most of those people used a Bausch \& Lomb ReNu (Rochester, NY) brand of contact lens solution, which included the solution MoistureLoc. The manufacturing company suspended sales voluntarily in these locations.

In March 2006, the Centers for Disease Control and Prevention in the United States received a report from an ophthalmologist of three cases of people who wore lenses who had Fusarium keratitis during the preceding two months. In the following months, more than 150 cases were identified in 33 US states and one US territory. All the people affected wore soft contact lenses.

Subsequent epidemiological investigation, which included (but was not limited to) a case-control study, found that people who had Fusarium keratitis were 13 times more likely to report using MoistureLoc than controls. All the tested samples of the solution were sterile and genotyping of Fusarium isolates showed great diversity, indicating that a common source during production was unlikely.

More than a third of the people with Fusarium keratitis required corneal transplantation. How MoistureLoc facilitates the occurrence of Fusarium keratitis remains unclear, but the product has been withdrawn from the markets worldwide. The authors recommend that people who wear soft contact lenses should follow the hygiene instructions more carefully.

JAMA 2006;296:953-63

\section{Outbreak of Marburg haemorrhagic fever in Congo sheds more light on the disease}

A report describes the laboratory findings, virus phylogeny, epidemiology, and clinical features of the outbreak of Marburg haemorrhagic fever in northeastern Democratic Republic of the Congo.
Between 1998 and 2000, more than 150 people fell ill with the disease. The outbreak had a case fatality rate of $83 \%$.

A filovirus, cousin to Ebola, the Marburg virus was first isolated and the Marburg haemorrhagic fever was first diagnosed in 1967 in Germany and Yugoslavia. Both countries had imported infected green monkeys from Uganda. For decades only sporadic cases in Africa were reported, until the outbreak in Congo and the more recent one in Angola in 2004 and 2005-the largest to date.

More than half of the people with the disease in Congo worked in an underground goldmine in the village of Durba. Less than a third of diseased miners reported having had contact with another infected person, compared with more than two thirds of people who did not work in a mine. Nine genetically distinct lineages of the virus were identified, and the end of the outbreak coincided with a flood in the mine.

The evidence suggests that the unknown reservoir of the virus often resides in habitats such as caves and mines. Researchers, however, have not been able to isolate the virus from the most incriminated species of animal-bats. Pathways of transmission to humans and other primates also remain unknown.

N Engl J Med 2006;355:909-19

\section{What works for preventing pressure ulcers?}

Pressure ulcers interfere with the recovery of patients from primary disease and cause substantial costs, but are often preventable. A systematic review looking at interventions for preventing pressure ulcers found 59 randomised controlled trials that included nearly 14000 patients.

Most trials studied interventions that targeted impaired mobility. Support surfaces with preventive effect included mattress overlays on operating tables, specialised foam overlays, and specialised sheepskin overlays. No overwhelming evidence showed that dynamic support surfaces, such as alternating pressure mattresses, low air loss beds, and air-fluidised mattresses, offered advantages over cheaper static support surfaces.

One trial showed that nutritional supplements significantly reduced the risk of pressure ulcers, but it is still unclear which micronutrients might be most beneficial. Topical care of sacral skin may 
be preventive because dryness of skin is a known risk factor for the development of pressure ulcers. No trials looked at simple moisturising of the skin, but one trial found a preventive effect in a topical hyperoxygenated fatty acid preparation.

Most protocols for prevention of pressure ulcers recommend frequent repositioning of the patient, but no trials looked at this intervention. It remains unclear whether some such strategies have advantages over others.

JAMA 2006;296:974-84

\section{Deep brain stimulation adds value to drug treatment of Parkinson's}

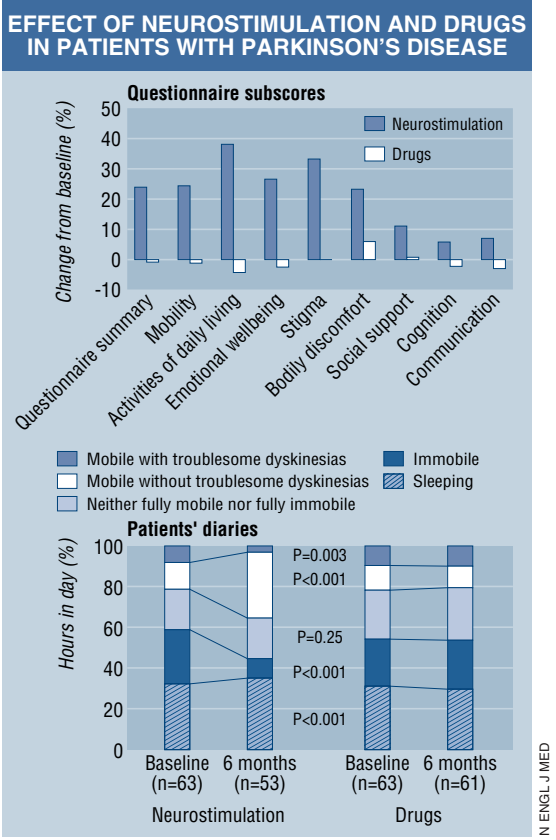

Does deep brain stimulation add value to the best drug regimen for patients aged $<75$ years with advanced Parkinson's disease and severe motor complications caused by treatment with levodopa? Deep brain stimulation of the subthalamic nucleus is achieved by implanting a permanent electrode and a pulse generator during bilateral stereotactic surgery under local anaesthesia.

This multicentre trial, which included 156 patients in a randomised pairs design and lasted for six months, measured motor functioning and overall quality of life. Any motor benefits in these patients might be outweighed by complications associated with the operation or neuropsychiatric adverse effects of stimulation. Despite this, the trial seems to show that, on the whole, neurostimulation does add value to best medical treatment alone.

People randomised to deep brain stimulation showed improvements in activities of daily living, emotional wellbeing, stigma, and bodily discomfort. No change was seen in cognition, mood, and overall psychiatric functioning. The brain stimulation group had less adverse events $(64 \%$ v $50 \%, \mathrm{P}=0.08)$, but a higher rate of serious adverse events $(13 \% v 4 \%$, $\mathrm{P}<0.04)$, including one death from intracerebral haemorrhage.

N Engl J Med 2006;355:896-908

\section{Treat DVT out of hospital with subcutaneous heparin}

Deep vein thrombosis is usually treated with intravenous infusion of unfractionated heparin with coagulation monitoring in hospital. The dose is constantly adjusted according to the measured activated partial thromboplastin time.

An open label trial of subcutaneous fixed dose unfractionated heparin versus subcutaneous low molecular weight heparin in more than 700 patients met the pre-specified non-inferiority margin, showing that neither regimen was significantly more effective nor safe. Unfractionated heparin was given by subcutaneous injection with an initial dose of 333 units per kilogram followed by a fixed dose of 250 units per kilogram every 12 hours. Low molecular weight heparin was given by subcutaneous injection in a dose of 100 units per kilogram every 12 hours. All patients also received warfarin over three months. More than two thirds of the patients in both groups were treated out of hospital.

The trial shows that monitoring of activated partial thromboplastin time may not be required for percutaneous unfractionated heparin, but a linked editorial (p 991) calls for caution before this is tested in bigger double blind trials. Although the efficacy and safety of the two regimens are similar, the editorial also poses a question: should patients with venous thromboembolism be treated with unfractionated heparin instead of low molecular weight heparin to save money? Based on the wholesale prices in the United States, costs for a six day course of treatment for a patient weighing $80 \mathrm{~kg}$ would be $\$ 712$ (£374, €553) for low molecular weight heparin and $\$ 37$ for unfractionated heparin. The trial has no bearing on the fact that activated partial thromboplastin time needs to be monitored in patients receiving infusions of unfractionated heparin.

\section{JAMA 2006;296:935-42}

\section{Celecoxib showed promise for prevention of colorectal adenomas but risks outweigh the benefits}

It is recommended that all patients who have had a colorectal adenoma removed undergo repeated colonoscopies every three to five years for secondary prevention of colorectal cancer. A drug that prevents adenomas and colorectal cancer could be

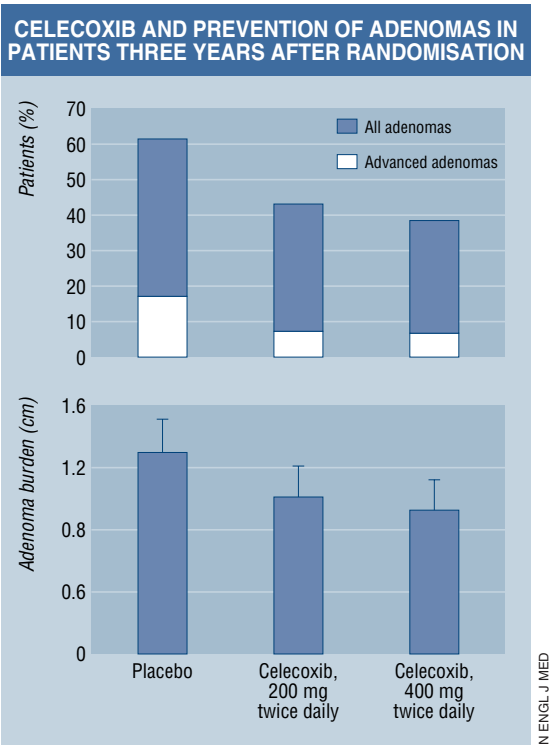

used to widen the interval between colonoscopies in these patients. Two randomised placebo controlled trials assessed the efficacy and safety of celecoxib (a cyclo-oxygenase-2 inhibitor) as such a chemopreventive agent. Together, the trials included more than 3300 people.

Both trials were stopped early after it became clear from these and other trials that celecoxib increased cardiovascular risk. The trials were similarly designed and differed mostly in the dosage of celecoxib. Participants randomised to celecoxib had a lower risk of adenoma being found in colonoscopies one year and three years after randomisation, compared with participants receiving placebo. For example, the risk at three years in people receiving $400 \mathrm{mg}$ of celecoxib was almost halved.

Although the efficacy outcomes were similar between the two trials, the rates of recorded adverse events differed. For example in one trial, the relative risk of serious cardiovascular events in people receiving $200 \mathrm{mg}$ of celecoxib twice daily compared with placebo was 2.6 (95\% CI 1.1 to 6.1). However, in the other trial, people receiving $400 \mathrm{mg}$ of celecoxib once daily had the corresponding relative risk of 1.3 (95\% CI 0.6 to 2.6). People randomised to $400 \mathrm{mg}$ twice daily had a 3.4-fold increased risk of serious cardiovascular events. Combined, the trials suggest about a doubled cardiovascular risk of celecoxib, compared with placebo.

A linked editorial (p 950) discusses how risks of celecoxib outweigh the potential benefits and suggests that the drug has no role as a chemopreventive agent in patients with non-familial colonic adenomas or in the general population.

N Engl J Med 2006;355:873-84, 885-95

Kristina Fister associate editor

kfister@bmj.com 